\title{
Orthopaedic Science
}

(C) The Japanese Orthopaedic Association

\section{Editorial}

\section{Research: intuition and logic}

\author{
Atsumasa Uchida \\ Department of Orthopedic Surgery, Mie University Postgraduate School of Medicine, 2-174 Edobashi, Tsu, Mie 514-8507, Japan
}

Research naturally must be supported by logic; however, excellent research results cannot be obtained through logicality alone. High-level research begins to become achievable only when research intuition is applied, and achievement in research that is highly appraised around the world provides logical support for ingenious original hypotheses. I suppose you have had the experience of feeling that you let a big fish escape because of the absence of that sense in your own research. I myself am one of those who have lamented the absence of "research sense." Bisphosphonate is currently one of the drugs that is being watched all over the world, and I was the first in Japan to investigate the effect of bisphosphonate on bone and cartilage metabolism. In an experiment in which bisphosphonate was added to cultured growth cartilage cells, matrix synthesis by the cells significantly increased. However, I remember that its range of effective concentrations was very narrow, and that when the range was exceeded, it had an inhibitory effect (Bone Metabolism 1978;11:261268). Convinced that for that reason it would be difficult to use as a drug, I turned my back on it completely. The subsequent development of bisphosphonate was remarkable, and I salute the research sense and efforts of the research group that promoted it.

It is now easily possible through journals and the Internet to have access to an enormous numbers of papers. However, determining whether their messages are based on facts or just someone's opinion largely depends on the reader's sensibility. These days, when research results are in such strong demand, it seems that a considerable amount of unsubstantiated information is being presented as though it were established fact. The main reason for this state of affairs is that investigators are lacking in basic ethics, but it is also

Offprint requests to: A. Uchida

Received: June 28, 2005 time to re-examine how scientific research should be supported.

The optimal approach to basic musculoskeletal research must also be considered in the middle of this Bone and Joint Decade. Of course, we must publish research data that outstrips that in other fields, but we must also seriously tackle such common diseases as frozen shoulder, tennis elbow, and muscle contusion. Research based on abundant intuition is required to obtain new results linked to prevention and the shortening of treatment that can be easily understood by general orthopedic surgeons and the general public, not just by investigators. In simple terms: intuition is greatly needed for research into frequently occurring diseases.

There are active Japanese orthopedic researchers in other countries. Intuition in research has also characterized the work that has given rise to their superb achievements in the tough environment abroad. Strengthening the liaison between orthopedic surgeons and basic researchers related to engineering and pharmaceutical manufacturing and extending the research circle is needed, but research intuition is also demanded to attract investigators in other fields.

Philosophers and historians of science have concluded that conflicts of ideas in the scientific community cannot be resolved on an entirely objective basis; rather, their resolution must involve subjective judgments - intuition and imagination, in other words. These subjective judgments involve the intellectual context of the work. In contrast to this view, many students believe that conflicts can be resolved in a completely objective manner. In addition, the distinctions between error, disagreement, and fraud are an important part of the public's response to scientific disagreements. The many disputes over evidence and interpretation must be analyzed in terms of the depth of the argument and so on, as well as the roles of intuition and imagination. 
Imagination is also very important in both undergraduate and postgraduate medical education. The same is true in research, because I think that what we truly want to convey to our successors is not knowledge and logic, but keen powers of observation, a tenacious capacity to think, and the ability to make sound judgments. I feel strongly that this is what is of paramount importance for physicians and investigators.

The 20th Japanese Orthopaedic Association Research Meeting will be held in Ise, Mie Prefecture, October 20 and 21, 2005, and wide-ranging educational and training lectures and symposia extending from basic musculoskeletal research to peripheral fields have been planned. Notably, we have also scheduled discussions on the privacy rule regarding human materials in clinical and basic research and on the proper approach to basic research that complies with research ethics guidelines. We will organize several symposia on topics of current interest in the field of orthopedics: regeneration of intervertebral discs, nerve regeneration, the molecular biology of musculoskeletal tumors, biomedical markers of arthritis, the biomechanics of ligament reconstruction, new biomaterials, basic research in common orthopedic diseases, cartilage regeneration, and mechanisms of cancer metastasis.

We hope that at the meeting a clear path will emerge for the pursuit of musculoskeletal science in the future. We also hope that young physicians, in addition to taking advantage of the meeting to absorb the latest knowledge in the field, will see it as a prime opportunity to get a feeling for the intuition of top-notch investigators. 